\title{
Water production rate of comet C/1997 H2 (SOHO) near perihelion
}

\author{
Salvatore Mancuso
}

\author{
Istituto Nazionale di Astrofisica, Osservatorio Astrofisico di Torino, Strada Osservatorio 20, 10025 Pino Torinese, Italy \\ e-mail: mancuso@oato.inaf.it
}

Received 18 March 2015 / Accepted 18 May 2015

\begin{abstract}
Comet C/1997 H2 is a non-Kreutz comet that was observed near perihelion at 0.137 AU on 1997 May 2 with the Ultraviolet Coronagraph Spectrometer (UVCS) aboard the Solar and Heliospheric Observatory (SOHO) satellite. The observed H I Lyman $\alpha$ radiance profile at $1215.67 \AA$, which was obtained during the crossing of the cometary coma through the UVCS slit positioned at $8.48 R_{\odot}$, was least-squares fitted to a Haser model with hydrogen atom velocities of $\sim 20 \mathrm{~km} \mathrm{~s}^{-1}$ and $\sim 8 \mathrm{~km} \mathrm{~s}^{-1}$ (from photodissociation of $\mathrm{H}_{2} \mathrm{O}$ and $\mathrm{OH}$, respectively). In order to obtain a better agreement between the observed $\mathrm{H}$ I Ly $\alpha$ radiance profile and the model, an additional population of low-velocity $\left(\sim 4 \mathrm{~km} \mathrm{~s}^{-1}\right)$ hydrogen atoms was required. This low-velocity component is attributed to thermalization of a substantial fraction $(\approx 30 \%)$ of fast hydrogen atoms within the cometary collision zone. The hydrogen production rate of comet $\mathrm{C} / 1997 \mathrm{H} 2$ for the crossing near perihelion corresponds to a water production rate of $Q_{\mathrm{H}_{2} \mathrm{O}}=6.64 \times 10^{28}$ molecules per second.
\end{abstract}

Key words. comets: individual: C/1997 H2 (SOHO) - ultraviolet: general

\section{Introduction}

Solar and Heliospheric Observatory (SOHO) instruments have observed about three thousand near-Sun comets since 1996, the vast majority of which are members of the Kreutz family of sungrazing comets. Most of the comets have been detected by the Large Angle Spectrometric Coronagraph (LASCO; Brueckner et al. 1995), which observes the white-light corona between about 2.3 and $32 R_{\odot}$, and by the Solar Wind ANisotropies (SWAN; Bertaux et al. 1995) instrument, a scanning imager that maps the full sky in $\mathrm{H}$ I Ly $\alpha$. The huge envelope of atomic hydrogen around the nucleus, or coma, is mainly produced by the UV photodissociation of cometary water $\left(\mathrm{H}_{2} \mathrm{O}\right.$ vapor from ice sublimation of the nucleus or surrounding grains) and its byproduct hydroxyl $(\mathrm{OH})$. Observations of the intensity profile of the resonantly scattered radiation illuminated by solar H I Ly $\alpha$ at 1215.67 $\AA$ can provide information on the column density of the atomic hydrogen coma as a function of distance from the cometary nucleus and thus yield an estimate of the water production rate. Since all other constituents in the coma are normally compared to water, it is important to obtain accurate evaluation of production rates.

Over the past two decades, several comets have also been spectroscopically detected by the UltraViolet Coronagraph Spectrometer (UVCS; Kohl et al. 1995) instrument onboard SOHO (Raymond et al. 1998, 2002; Uzzo et al. 2001; Povich et al. 2003; Bemporad et al. 2005, 2007; Ciaravella et al. 2010; Giordano et al. 2015). In this Letter, we report the water production rate at perihelion of a non-Kreutz comet observed by UVCS, comet C/1997 H2 (Stezelberger et al. 1997), as determined from the $\mathrm{H}$ I Ly $\alpha$ intensity profile obtained when the comet was at a distance of $\sim 0.14$ AU from the Sun. The overall contribution of neutral hydrogen atoms created by photodissociation processes from $\mathrm{H}_{2} \mathrm{O}\left(\sim 20 \mathrm{~km} \mathrm{~s}^{-1}\right)$ and $\mathrm{OH}\left(\sim 8 \mathrm{~km} \mathrm{~s}^{-1}\right)$ is estimated by fitting a Haser model (Haser 1957) to the observed H I Ly $\alpha$ radiance profile. To obtain a better agreement between

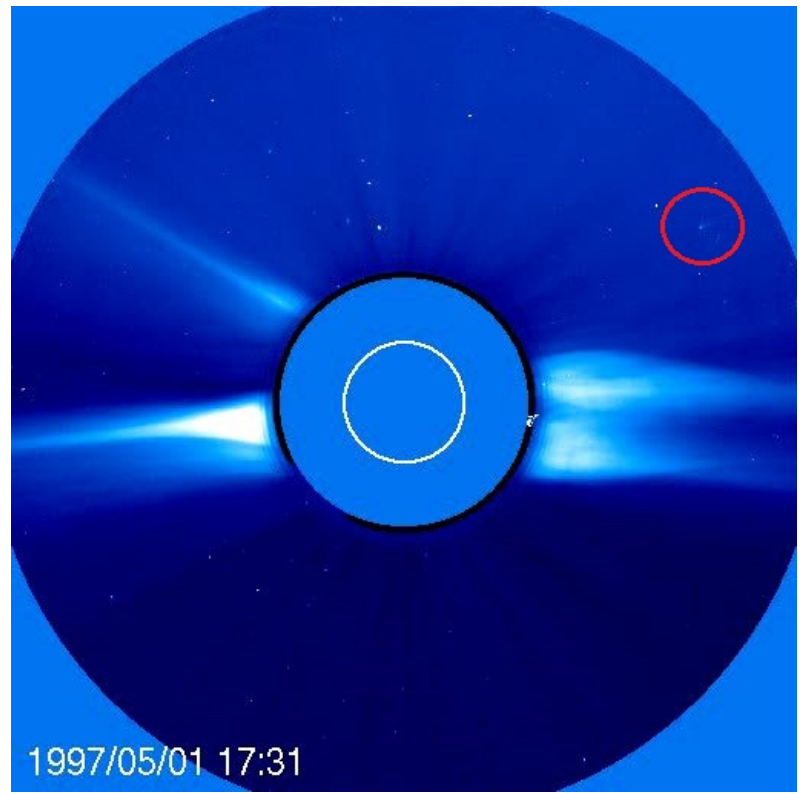

Fig. 1. LASCO C2 image of the solar corona on 1997 May 1 at 17:31 UT showing comet C/1997 H2 approaching the Sun from northwest (inside red circle).

the observed radiance profile and the model, however, an additional fraction of low-velocity hydrogen atoms $\left(\sim 4 \mathrm{~km} \mathrm{~s}^{-1}\right)$ is required. This low-velocity component is attributed to thermalization of fast hydrogen atoms within the collision zone of the comet.

\section{Observations}

In Fig. 1, we show the visual appearance of comet C/1997 H2 as seen in white light by the LASCO C2 coronagraph. The comet approached the Sun from the northwest quadrant at a position angle (measured counterclockwise from celestial north) of $296.2^{\circ}$ 
projected onto the plane of the sky. Detections were first made with the LASCO C3 coronagraph on 1997 April 29, at $31.43 R_{\odot}$ from the Sun's center. According to the orbital data, given in the Minor Planet Electronic Circular (MPEC) 1997-J05, the comet reached a perihelion distance of $q=0.137 \mathrm{AU}$ at $t_{p}=20: 00 \mathrm{UT}$ on 1997 May 2. UVCS is a long-slit UV spectrograph consisting of two spectrometric channels (the OVI and LYA channels) for the observation of spectral lines in the UV range with an instantaneous field of view of $42^{\prime}$ tangent to the limb of the Sun with nominal spatial resolution of $7^{\prime \prime}$ per pixel $(\sim 5000 \mathrm{~km}$ at the Sun). The UVCS telescope mirror and instrument rotation mechanisms can point the spectrograph slits in the radial direction to observe the entire corona at heliocentric distances between about 1.5 and $10 R_{\odot}$. The position of the UVCS slit for the observations analyzed in this work was at a projected height of $8.48 R_{\odot}$ and a roll angle of $110^{\circ}$ counterclockwise from north pole. At this slit position, the UVCS observations, composed of a total of 83 exposures of $200 \mathrm{~s}$ each, started at 20:11 UT on 1997 May 2 and ended at 01:08 UT on the next day. The comet nucleus entered the slit at the exposure taken between 23:15.4 and 23:18.7 UT. Over the available ranges, only the $\operatorname{Ly} \alpha, \operatorname{Ly} \beta$ and Ly $\gamma$ lines were detected. The H I Ly $\alpha 1215.67 \AA$ line was well observed spectroscopically. Significant emission in the $\mathrm{H}$ I Ly $\beta$ $1025.72 \AA$ line and weak emission in H I Ly $\gamma 972.02 \AA$ were also observed in the hydrogen inner coma. Figure 2 shows the H I Ly $\alpha 1215.67 \AA$ image of comet C/1997 H2, reconstructed from UVCS observations. Details and thorough spectroscopic analysis of the complete set of observations obtained for comet C/1997 H2 by UVCS from 1997 May 1 to May 3 are out of the purpose of this Letter and will be presented elsewhere.

\section{Data analysis and modeling}

The observed cometary H I Ly $\alpha$ emission may arise from collisional excitation with thermal electrons and/or from resonant scattering of the H I Ly $\alpha$ at $1215.67 \AA$ emitted by the solar chromosphere. To understand its origin, we estimated the observed ratio between the $\operatorname{Ly} \alpha$ and $\operatorname{Ly} \beta$ spectral line intensities. The intensity ratio $\operatorname{Ly} \alpha / \operatorname{Ly} \beta$ is about 400 around the nucleus, roughly doubling up at a distance of $5 \times 10^{4} \mathrm{~km}$ from the nucleus. Collisional excitation at the high electron temperatures typical of the solar wind would produce a ratio close to 8 (Raymond et al. 1998), while scattering of emission-line photons from the solar disk produces a much higher ratio (because of the very high $\operatorname{Ly} \alpha / \operatorname{Ly} \beta$ ratio of the chromospheric emission) of about 910 (Raymond et al. 1997). Therefore, the observed Ly $\alpha$ in the extended coma is mostly attributable to resonant scattering from neutral hydrogen atoms, while a partial contribution from collisional excitation is possible at a few $\times 10^{4} \mathrm{~km}$ from the nucleus (e.g., Raymond et al. 2002). As suggested by the Referee of this Letter, the $\operatorname{Ly} \alpha / \operatorname{Ly} \beta$ ratio could also indicate the emission of $\operatorname{Ly} \alpha$ and $\mathrm{Ly} \beta$ by electron excitation of $\mathrm{H}_{2} \mathrm{O}$ molecules. Electrons produced by the ionization of $\mathrm{H}_{2} \mathrm{O}$ molecules have enough energy to dissociate the $\mathrm{H}_{2} \mathrm{O}$ molecule and generate $\mathrm{H}$ atoms in an excited state, thus producing emission in both $\operatorname{Ly} \alpha$ and $\operatorname{Ly} \beta$ spectral lines with a $\operatorname{Ly} \alpha / \operatorname{Ly} \beta$ ratio that is much lower than for resonance scattering from solar photons, as is actually observed in this observation near the nucleus.

The observed Ly $\alpha$ line emission stems from a superposition of the cometary signal, foreground coronal emission, interplanetary emission and detector dark counts. To identify the cometary signal, the average emission over several exposures taken before the cometary coma entered the UVCS field of view was

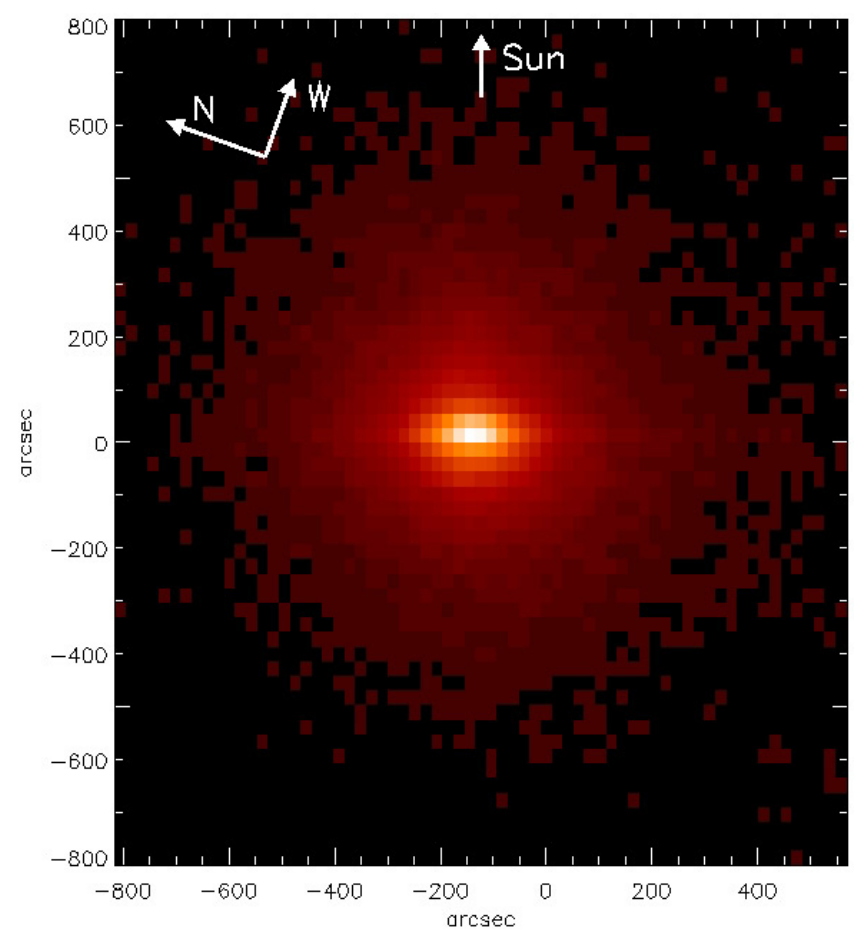

Fig. 2. Reconstructed image of the H I Ly $\alpha$ light from the comet C/1997 $\mathrm{H} 2$ as observed at $8.48 R_{\odot}$. The horizontal axis represents the position (expressed in arcsec) along the UVCS slit. Each bin in the vertical direction is given by the product of the single exposure time and the comet velocity in the plane of the sky. Courtesy of S. Giordano.

subtracted. The data were then calibrated using previously generated calibration files that consider such factors as the internal occulter setting and the grating position (Gardner et al. 2002).

The hydrogen atoms that scatter $\mathrm{H}$ I Ly $\alpha$ radiation are mainly formed by dissociation of molecules outgassed from the cometary nucleus. Hydrogen is produced primarily by photodissociation of water through the following reactions:

$$
\mathrm{H}_{2} \mathrm{O}+h v \rightarrow \mathrm{OH}+\mathrm{H}
$$

$\mathrm{OH}+h v \rightarrow \mathrm{O}+\mathrm{H}$.

The cometary outgassing rate of water vapor $Q_{\mathrm{H}_{2} \mathrm{O}}$ can be estimated from the observed Ly $\alpha$ radiance distribution by assuming that the photodissociation of water outside the collision sphere is the only noteworthy process. The production rate, $Q_{\mathrm{H}_{2} \mathrm{O}}$, is assumed to be constant (stationary model) and isotropic. All particles are assumed to have a radial velocity, and their distribution is spherically symmetric (Haser 1957). The column densities of the two $\mathrm{H}$ populations produced by photodissociation of water at a distance $r$ from the nucleus are obtained by integration along the line of sight. If $N_{\mathrm{H}_{1}}$ and $N_{\mathrm{H}_{2}}$ represent the column densities coming, respectively, from the first photodissociation process (Eq. (1)) and from the second photodissociation process (Eq. (2)), they can be expressed (e.g., Mäkinen et al. 2001) as

$$
\begin{aligned}
& N_{\mathrm{H}_{1}}(r)=\frac{2 Q_{\mathrm{H}_{2} \mathrm{O}}}{4 \pi v_{\mathrm{H}_{1}} r} \frac{\gamma_{\mathrm{H}_{2} \mathrm{O}}}{\gamma_{\mathrm{H}_{1}}-\gamma_{\mathrm{H}_{2} \mathrm{O}}}\left[f\left(\gamma_{\mathrm{H}_{2} \mathrm{O}} r\right)-f\left(\gamma_{\mathrm{H}_{1}} r\right)\right], \\
& N_{\mathrm{H}_{2}}(r)=\frac{2 Q_{\mathrm{H}_{2} \mathrm{O}}}{4 \pi v_{\mathrm{H}_{2}} r} \gamma_{\mathrm{H}_{2} \mathrm{O}} \gamma_{\mathrm{OH}}\left[\frac{f\left(\gamma_{\mathrm{H}_{2} \mathrm{O}} r\right)}{\left(\gamma_{\mathrm{H}_{2}}-\gamma_{\mathrm{H}_{2} \mathrm{O}}\right)\left(\gamma_{\mathrm{OH}}-\gamma_{\mathrm{H}_{2} \mathrm{O}}\right)}\right. \\
& \left.-\frac{f\left(\gamma_{\mathrm{OH}} r\right)}{\left(\gamma_{\mathrm{OH}}-\gamma_{\mathrm{H}_{2} \mathrm{O}}\right)\left(\gamma_{\mathrm{H}_{2}}-\gamma_{\mathrm{OH}}\right)}+\frac{f\left(\gamma_{\mathrm{H}_{2}} r\right)}{\left(\gamma_{\mathrm{H}_{2}}-\gamma_{\mathrm{H}_{2} \mathrm{O}}\right)\left(\gamma_{\mathrm{H}_{2}}-\gamma_{\mathrm{OH}}\right)}\right] .
\end{aligned}
$$

In the above equations, $\gamma_{i}$ and $v_{i}\left(i=\mathrm{H}_{2} \mathrm{O}, \mathrm{OH}, \mathrm{H}_{1}, \mathrm{H}_{2}\right)$ are the inverse scale lengths $\left(L_{i}=\gamma_{i}^{-1}\right)$ and the radial velocities of the 
respective particle populations, $r$ is the shortest distance between the column and the nucleus and $f(x) \equiv \int_{0}^{\pi / 2} \exp (-x / \cos \alpha) \mathrm{d} \alpha$. Typical hydrogen ejection velocities for the two different populations are $v_{\mathrm{H}_{1}}=20 \mathrm{~km} \mathrm{~s}^{-1}$ and $v_{\mathrm{H}_{2}}=8 \mathrm{~km} \mathrm{~s}^{-1}$ (e.g., Combi \& Smyth 1988; Bertaux et al. 1998). The scale lengths of the hydrogen-producing processes at $1 \mathrm{AU}$ are $L_{\mathrm{H}_{2} \mathrm{O}}(1 \mathrm{AU})=$ $8.2 \times 10^{4} \mathrm{~km}$ and $L_{\mathrm{OH}}(1 \mathrm{AU})=2.25 \times 10^{5} \mathrm{~km}$ (Mäkinen et al. 2001), while the scale lengths for the two hydrogen components are given by the product of the respective outgassing velocities and their lifetime $\tau_{\mathrm{H}}$.

The total intensity, $I_{\mathrm{Ly} \alpha}$ along the line of sight is given by

$I_{\mathrm{Ly} \alpha}=I_{\mathrm{Ly} \alpha, \mathrm{H}_{1}}+I_{\mathrm{Ly} \alpha, \mathrm{H}_{2}}$,

where

$I_{\text {Ly } \alpha, j}=\frac{g\left(d_{\text {comet }}\right) N_{j}}{4 \pi} \mathrm{e}^{-\tau_{j}}$,

are, respectively, the contributions from hydrogen atoms given by Eqs. (1) and (2), $N_{j}$ (with $j=\mathrm{H}_{1}, \mathrm{H}_{2}$ ) is the column density of the scattering $\mathrm{H}$ atoms, and

$g\left(d_{\text {comet }}\right)=\frac{\pi e^{2}}{m_{\mathrm{e}} c} f_{12} \frac{\lambda_{\mathrm{Ly} \alpha}^{2}}{c} \frac{\pi F_{0}}{d_{\text {comet }}^{2}} p_{\phi}$

is the $g$ factor, that is, the number of Ly $\alpha$ photons per second fluorescently produced by absorption of chromospheric Ly $\alpha$ photons by each $\mathrm{H}$ atom in the line of sight at a distance $d=d_{\text {comet }}$ from the Sun. In Eq. (7), $\frac{\pi e^{2}}{m_{\mathrm{e}} c} f_{12}$ is the frequency-integrated cross section of the transition $\left(f_{12}=0.416\right.$ being the oscillator strength) that multiplied by the factor $\lambda_{\mathrm{Ly} \alpha}^{2} / c$ yields the wavelength integrated cross section; $\pi F_{0}$ is the solar Ly $\alpha$ flux per unit wavelength interval at $1 \mathrm{AU}$ at the Doppler-shifted wavelength corresponding to the heliocentric radial velocity of the comet; $p_{\phi}$ is the probability that in a scattering process a photon changes its direction of an angle $\phi$ relative to the initial direction. A $g$ factor value $g\left(d_{\text {comet }}\right)=0.0953$ photons s ${ }^{-1}$ was determined from daily average values obtained by the SOLar Stellar Irradiance Comparison Experiment (SOLSTICE) onboard NASA's Upper Atmosphere Research Satellite (UARS), which measures the solar UV radiance. The estimate was corrected for the solar rotation difference between the face of the Sun seen by the instrument and that seen by the comet and for the Doppler shift in the H I Ly $\alpha$ line profile caused by the heliocentric radial velocity of the comet $\left(36 \mathrm{~km} \mathrm{~s}^{-1}\right.$, as calculated from the given orbital parameters) toward the Sun, on the basis of the solar irradiation profile around the Ly $\alpha$ line as given by Lemaire et al. (1998). We also took the optical thickness for each component $\left(=N_{j} \sigma_{j}\right)$ into account with $\sigma_{j}$ representing the scattering cross sections that depend on the velocities of the different populations $v_{\mathrm{H}_{j}}$. Optical depth effects have been included because they can be important near the nucleus where a stronger contribution of the collisional component in the Ly $\alpha$ emission is expected.

Neutral hydrogen atoms released by a comet are lost from the coma through a combination of photoionization by the solar $\mathrm{UV}$ radiation, impact ionization by solar wind electrons (Combi et al. 1986) and charge exchange with solar wind protons (Keller 1973; Bertaux et al. 1973). For H atoms released by the comet into the solar wind, their lifetime, $\tau_{\mathrm{H}}$, has been estimated to be in the range of $1-2 \times 10^{6} \mathrm{~s}$ at $1 \mathrm{AU}$, varying with the square of the heliocentric distance (e.g., Combi et al. 2005). The actual value, however, depends critically on several factors, such as the time and location, the solar wind density, velocity, electron temperature, the solar UV radiation flux, and the heliographic latitude.

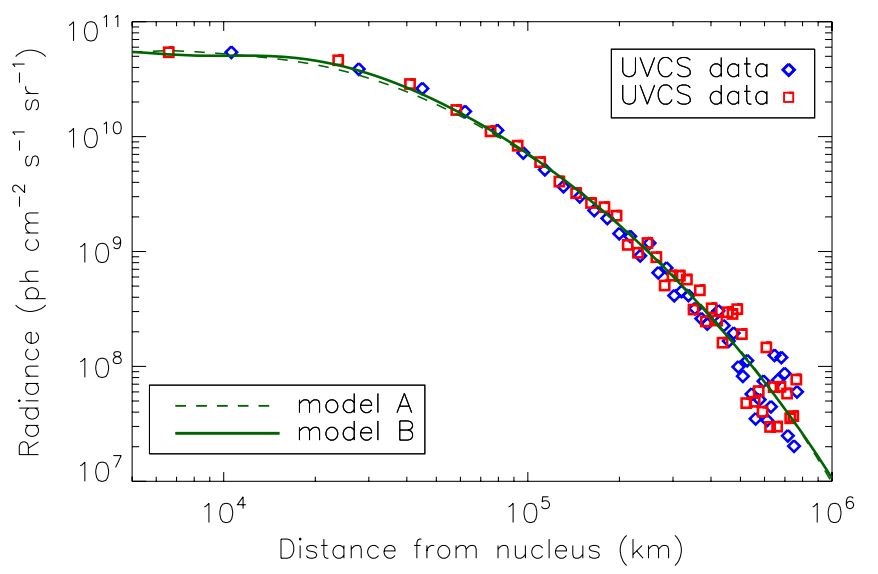

Fig. 3. H I Ly $\alpha$ radiance profile along the UVCS slit from the exposure containing the nucleus taken near perihelion (red squares and blue diamonds represent data taken north and south of the cometary nucleus). The green dashed curve is a model (Model A) obtained by considering two hydrogen atom populations $\left(v_{\mathrm{H}_{1}}=20 \mathrm{~km} \mathrm{~s}^{-1}, v_{\mathrm{H}_{2}}=8 \mathrm{~km} \mathrm{~s}^{-1}\right)$ with $Q_{\mathrm{H}_{2} \mathrm{O}}$ and $\tau_{\mathrm{H}}$ left as free parameters. The green solid curve is a model (Model B) obtained by adding the contribution of a thermalized hydrogen atom population $\left(v_{\mathrm{H}_{3}}=4 \mathrm{~km} \mathrm{~s}^{-1}\right)$. See the text for details.

Instead of assuming a fixed value for $\tau_{\mathrm{H}}$, we leave this atomic property as a free parameter, together with the unknown outgassing rate, $Q_{\mathrm{H}_{2} \mathrm{O}}$, to be solved by compariing the coma model with observations.

The above model (Model A) was applied and compared to the data obtained with the analysis of the Ly $\alpha$ radiance profile along the UVCS slit from the exposure containing the nucleus. The best-fit to the Ly $\alpha$ radiance profile observed on 1997 May 2 for the crossing at 23:17 UT is shown in Fig. 3 as a dashed line, superposed on the data. The model was obtained through a least-squares approach, yielding a water production rate of $Q_{\mathrm{H}_{2} \mathrm{O}}=7.39 \times 10^{28}$ molecules s${ }^{-1}$ and a hydrogen atom lifetime of $\tau_{\mathrm{H}}=0.85 \times 10^{6} \mathrm{~s}$ at $1 \mathrm{AU}$ as best-fitting parameters. Although Model A fits the UVCS data quite well (the different symbols in Fig. 3 specify radiance estimate north and south of the cometary nucleus), it underestimates by about $5 \%-10 \%$ the observed radiance between about $1-5 \times 10^{4} \mathrm{~km}$ from the nucleus. Moreover, the hydrogen atom lifetime is also underestimated, falling below the previously mentioned range of $1-2 \times 10^{6} \mathrm{~s}$ at $1 \mathrm{AU}$.

We note that the Haser model assumes a simple radial outflow of the daughter species once they are created, without considering the non-radial motion of the radicals produced after photodissociation or recombinative dissociation of the parent molecules outside the collision zone. A more realistic vectorial model, which assumes isotropic ejection of daughter species following photodissociation of the parent molecule, was introduced by Festou (1981). Both models are frequently used to determine the production rates of the observed daughter species, although neither the Haser nor the vectorial model include any collision effects. As pointed out by the Referee of this Letter, however, the vectorial model is flatter at the center than the Haser model so that its application would probably increase the discrepancy between the model and the UVCS data.

The approximation of two hydrogen atom populations with very narrow velocity distributions is probably reasonable only for small comets, for which most hydrogen atoms are produced outside the collision zone. In fact, Haser-like models obtained with a $\mathrm{H}$-atom distribution consistent with $\mathrm{H}_{2} \mathrm{O}$ and $\mathrm{OH}$ photodissociation yield reasonable results mostly for heliocentric 
distances $>0.8 \mathrm{AU}$ and gas production rates $<$ few $\times 10^{28} \mathrm{~s}^{-1}$, where thermalization of suprathermal $\mathrm{H}$ atoms can be ignored (Combi et al. 2005). For comets with higher gas production rates, the effect of collisions could redistribute a significant fraction of the high velocity atoms to lower kinetic energy, with significant pile-up at velocities $\lesssim 4 \mathrm{~km} \mathrm{~s}^{-1}$ (e.g., Combi \& Smith 1988). To obtain a better fit to the observed Ly $\alpha$ radiance profile of comet $\mathrm{C} / 1997 \mathrm{H} 2$, a low-velocity component is thus probably required. This low-velocity component could result from thermalization of fast hydrogen atoms within the collision zone.

The effectiveness of the thermalization depends on the number of atoms produced within the region of the coma, where collisions are important, and on temperature. To estimate the expected amount of this low-velocity component, we calculated the fraction of atoms produced within the cometary collision zone (see, e.g., McCoy et al. 1992). The radius of the collision zone, $R_{\mathrm{c}}$, is obtained by imposing $\int_{R_{\mathrm{c}}}^{\infty} n_{\mathrm{H}_{2} \mathrm{O}} \sigma \mathrm{d} r=1$, where $\sigma$ is the collisional cross section and $n_{\mathrm{H}_{2} \mathrm{O}}\left(=Q_{\mathrm{H}_{2} \mathrm{O}} / 4 \pi r^{2} v_{\mathrm{H}_{2} \mathrm{O}}\right)$ the water number density. Substituting this into the above integral and solving for $R_{\mathrm{c}}$, we estimate $R_{\mathrm{c}} \approx 1200 \mathrm{~km}$ for a cross section $\sigma \approx 2 \times 10^{-15} \mathrm{~cm}^{2}$ and a typical molecular outflow velocity $v_{\mathrm{H}_{2} \mathrm{O}} \approx 1 \mathrm{~km} \mathrm{~s}^{-1}$. The scale lengths for photodissociation of water at $d=0.137 \mathrm{AU}$ is thus comparable $(\sim 1500 \mathrm{~km})$ in magnitude to the collision distance of water molecules at this distance from the Sun.

Because of thermalization, a considerable fraction of the high-speed $\left(\sim 20 \mathrm{~km} \mathrm{~s}^{-1}\right)$ atomic $\mathrm{H}$ generated by the comet will thus be slowed down to $v \lesssim 4 \mathrm{~km} \mathrm{~s}^{-1}$ while the $\sim 8 \mathrm{~km} \mathrm{~s}^{-1}$ component from the $\mathrm{OH}$ radicals would probably survive in large part (see, e.g., Combi \& Smith 1988). This new situation can be approximated with a model (Model B) similar to the previous one that does, however, include a third component with speed $v_{\mathrm{H}_{3}}$, left as a free parameter, accounting for the broad thermalized component at low velocities. (We only considered integer values for $v_{\mathrm{H}_{3}}$ varying between 1 to $6 \mathrm{~km} \mathrm{~s}^{-1}$.)

As in the previous case, this model was applied to leastsquare fit the radiance of the $\mathrm{H} \mathrm{I} \operatorname{Ly} \alpha$ as observed by UVCS in the exposure where the cometary nucleus entered the slit, leaving the outgassing rate $Q_{\mathrm{H}_{2} \mathrm{O}}$, the hydrogen lifetime $\tau_{\mathrm{H}}$ at $1 \mathrm{AU}$, and the speed of the low-velocity component as free parameters. The weighting of these two components has also been left a free parameter in our model since the amount of thermalized high-speed hydrogen atoms is uncertain. As shown in Fig. 3, an outstanding fit to the observed $\operatorname{Ly} \alpha$ radiance profile was obtained for Model B with best-fitting parameters $Q_{\mathrm{H}_{2} \mathrm{O}}=$ $6.64 \times 10^{28}$ molecules s ${ }^{-1}$ and $\tau_{\mathrm{H}}=0.99 \times 10^{6} \mathrm{~s}$, together with the addition of a further low-speed component of $4 \mathrm{~km} \mathrm{~s}^{-1}$ attributed to thermalization of fast hydrogen atoms within the collision zone. Weights of 0.72 and 0.28 for the highest $\left(\sim 20 \mathrm{~km} \mathrm{~s}^{-1}\right)$ and lowest $\left(\sim 4 \mathrm{~km} \mathrm{~s}^{-1}\right)$ speed components, respectively, have been found to better fit the data, showing that a significant fraction of the high speed $\mathrm{H}$ atoms have been thermalized.

Finally, we point out that the nucleus of this comet is probably small in view of its relatively modest water production rate and the short distance from the Sun at which it was observed by UVCS. Approximating the cometary nucleus as a sphere, we can provide a rough estimate of its equivalent radius (e.g., Giordano et al. 2015) as $r_{\mathrm{eq}}=\sqrt{Q_{\mathrm{H}_{2} \mathrm{O}} L /\left[\pi F_{d}(1-A) N_{\mathrm{A}}\right]}$, where $L=4.81 \times 10^{11} \mathrm{erg} \mathrm{mol}^{-1}$ is the latent heat of sublimation of ice, $A=0.06$ the cometary albedo, $F_{d}$ the solar flux scaled to the cometary heliocentric distance and $N_{\mathrm{A}}$ the Avogadro's number.
Given the outgassing rate estimated in this work, the above relationship, which only holds in the absence of unobserved fragmentation events, yields $r_{\mathrm{eq}}=160 \mathrm{~m}$.

\section{Summary and conclusions}

In this work, we analyzed the $\mathrm{H}_{\mathrm{I}} \mathrm{Ly} \alpha$ emission at $1215.67 \AA$ from the extended coma of comet C/1997 H2 that was observed near perihelion at $0.137 \mathrm{AU}$ with the UVCS instrument onboard SOHO. The observed H I Ly $\alpha$ radiance profile of the cometary coma, mainly produced by the water dissociation chain, was compared with a Haser model with hydrogen atom velocities of $\sim 20 \mathrm{~km} \mathrm{~s}^{-1}$ and $\sim 8 \mathrm{~km} \mathrm{~s}^{-1}$ (from photodissociation of $\mathrm{H}_{2} \mathrm{O}$ and $\mathrm{OH}$, respectively). To obtain a better agreement with the UVCS H I Ly $\alpha$ observations in a range between about 1 and $5 \times 10^{4} \mathrm{~km}$ from the nucleus, a further low-velocity component of $4 \mathrm{~km} \mathrm{~s}^{-1}$ was, however, required. By taking the contribution from this additional slower component into account, the hydrogen production rate for the crossing near perihelion corresponds to a cometary water production rate of $Q_{\mathrm{H}_{2} \mathrm{O}}=$ $6.64 \times 10^{28}$ molecules $\mathrm{s}^{-1}$ and a hydrogen atom lifetime at $1 \mathrm{AU}$ of $\tau_{\mathrm{H}} \approx 1.0 \times 10^{6} \mathrm{~s}$. The $\sim 4 \mathrm{~km} \mathrm{~s}^{-1}$ low-velocity component is attributed to thermalization of a substantial fraction $(\approx 30 \%)$ of fast hydrogen atoms within the cometary collision zone.

Acknowledgements. UVCS is the result of a collaborative effort between NASA and ASI, with a Swiss participation. SOHO is a mission of international cooperation between ESA and NASA. The author wishes to express his thanks to the anonymous referee for the very helpful comments and suggestions that significantly improved the paper and to S. Giordano for producing Fig. 2 in this paper and for his valuable comments. This work, made possible by the UVCS/SOHO instrument and Flight Operations team, has been originally suggested and encouraged by E. Antonucci, lead observer for this particular UVCS observation.

\section{References}

Bemporad, A., Poletto, G., Raymond, J. C., \& Giordano S. 2007, Planet. Space Sci., 55, 1021

Bemporad, A., Poletto, G., Raymond, J. C., et al. 2005, ApJ, 620, 523

Bertaux, J. L., Blamont, J. E., \& Festou, M. 1973, A\&A, 25, 415

Bertaux, J. L., Kyrola, E., Quémerais, E., et al. 1995, Sol. Phys., 162, 403

Bertaux, J. L., Costa, J., Quémerais, E., et al. 1998, Planet. Space Sci., 46, 555

Brueckner, G., Howard, R., Koomen, M., et al. 1995, Sol. Phys., 162, 357

Ciaravella, A., Raymond, J. C., \& Giordano S. 2010, ApJ, 713, L69

Combi, M. R., \& Smyth, W. H. 1988, ApJ, 327, 1044

Combi, M. R., Stewart, A. I. F., \& Smyth, W. H. 1986, Geophys. Res. Lett., 13, 385

Combi, M. R., Mäkinen, J. T. T., Bertaux, J.-L., \& Quemerais, E. 2005, Icarus, 177,228

Festou, M. C. 1981, A\&A, 95, 69

Gardner, L. D., Smith, P. L., Kohl, J. L., et al. 2002, in The Radiometric Calibration of SOHO, eds. A. Pauluhn, M. C. E. Huber, \& R. von Steiger (Noordwijk: ESA), 161

Giordano, S., Raymond, J. C., Lamy, P., Uzzo, M., \& Dobrzycka, D. 2015, ApJ, 798,47

Haser, L. 1957, Bull. Class Sci. Acad. Roy. Belgique, 43, 740

Keller, H. U. 1973, A\&A, 27, 51

Kohl, J. L., Esser, R., Gardner, L. D., et al. 1995, Sol. Phys., 162, 313

Lemaire, P., Emerich, C., Curdt, W., et al. 1998, A\&A, 334, 1095

Mäkinen, J. T. T., Bertaux, J.-L., Pulkkinen, T. I., et al. 2001, A\&A, 368, 292

McCoy, R. P., Meier, R. R., Keller, H. U., Opal, C. B., \& Carruthers, G. R. 1992, A\&A, 258, 555

Povich, M. S., Raymond, J. C., Jones, G. H., et al. 2003, Science, 302, 1949

Raymond, J. C., Kohl, J. L., Noci, G., et al. 1997, Sol. Phys., 175, 654

Raymond, J. C., Fineschi, S., Smith, P. L., et al. 1998, ApJ, 508, 410

Raymond, J. C., Uzzo, M., Ko, Y.-K., et al. 2002, ApJ, 564, 1054

Stezelberger, S., St. Cyr, C., Williams, G. V., \& Marsden, B. G. 1997, Minor

Planet Electronic Circulars, 5

Uzzo, M., Raymond, J. C., Biesecker, D. A., et al., 2001, ApJ, 558, 403 